\title{
UNA REVISIÓN A LA REGLAMENTACIÓN E INCENTIVOS DE LAS ENERGÍAS RENOVABLES EN COLOMBIA*
}

\author{
DIANA CAROLINA ORTIZ MOTTA**, JAVIER SABOGAL AGUILAR ${ }^{* * * * *} \&$ ENRIQUE HURTADO AGUIRRE ${ }^{* * * * * *}$ \\ UNIVERSIDAD MILITAR NUEVA GRANADA
}

Recibido/ Received/ Recebido: 03/02/2012 - Aceptado/ Accepted / Aprovado: 15/09/2012

\begin{abstract}
Resumen
El documento analiza las estrategias de: promoción, producción, desarrollo o adaptación de las energías renovables en el país. El desarrollo de esta investigación partió del actual Plan Energético Nacional en lo referente a los lineamientos en materia de política energética. De igual manera se retomaron los programas, las leyes, los planes y los decretos relacionados con la planeación, ejecución y operación de los proyectos energéticos. La investigación realizada permitió concluir que existen leyes e incentivos, principalmente de carácter tributario, que promueven el desarrollo de las energías renovables. Sin embargo, en el país aún se requiere profundizar en estudios que generen cuantificaciones continuas del potencial de implementación de fuentes no convencionales de energía, de manera que se mejoren las políticas energéticas, permitiendo establecer estrategias de largo plazo que involucren los componentes sociales y ambientales.
\end{abstract}

Palabras clave: Energías renovables, Fuentes no convencionales de energía, Regulación e incentivos en energías renovables.

\section{A REVIEW TO REGULATION AND INCENTIVES OF RENEWABLE ENERGIES IN COLOMBIA}

\begin{abstract}
This article analyses the strategies of: promotion, production, development or adaptation of renewable energies in the country. The development of this research began with the current National Energetic Plan regarding to guidelines of energetic policy. Also, the programs, laws, plans and decrees related to planning, execution and operation of energetic projects were reviewed. This research made possible to conclude that there are laws and incentives, manly tributary that promote development of renewable energies. However, it is necessary to study deeper continuous quantifications of the implementation potential of non-conventional energy sources in the country, in order to improve energetic policies, and to allow establishing long term strategies that involve social and environmental components.
\end{abstract}

Keywords: Renewable energies, Non-conventional energy sources, Regulation and incentives in renewable energies.

* Proyecto desarrollado en el Grupo de Estudios en Ciencias Económicas (CIE) de la Universidad Militar Nueva Granada. Resultado del Proyecto de Investigación ECO 718, denominado: "Procesos de Transferencia Tecnológica en Proyectos de Mecanismo de Desarrollo Limpio (MDL) del Sector de Generación Hidroeléctrica en Colombia”. Financiado la Vicerrectoría de Investigaciones de la UMNG.

** Economista de la Universidad Militar Nueva Granada y Estudiante de Maestría en Estudios y Gestión del Desarrollo de la Universidad de la Salle. Joven Investigadora Grupo CIE, Facultad de Ciencias Económicas, Universidad Militar Nueva Granada, Bogotá, Colombia. Correo electrónico: diana.ortiz@unimilitar.edu.co

**** Doctor (c) en Ciencias Económicas, Maestría en Gestión Ambiental y Administrador de la Universidad Nacional de Colombia. Docente e Investigador tiempo completo Grupo CIE, Facultad de Ciencias Económicas, Universidad Militar Nueva Granada, Bogotá, Colombia. Correo electrónico: javiersabogala@gmail.com

**:**: Magister en Administración y Administrador de la Universidad Nacional de Colombia. Docente e Investigador tiempo completo Grupo CIE, Facultad de Ciencias Económicas, Universidad Militar Nueva Granada, Bogotá, Colombia. Correo electrónico: enrique. hurtado@unimilitar.edu.co 


\title{
UMA REVISÃO DA REGULAMENTAÇÃO E DOS INCENTIVOS DAS ENERGIAS RENOVÁVEIS NA COLÔMBIA
}

\begin{abstract}
Resumo
O documento analisa as estratégias de promoção, produção, desenvolvimento ou adaptação das energias renováveis no país. O desenvolvimento desta pesquisa partiu do atual Plano Energético Nacional no que se refere aos lineamentos em matéria de política energética. Do mesmo modo se retomaram os programas, as leis, os planos e os decretos relacionados com o planejamento, execução e operação dos projetos energéticos. A pesquisa realizada permitiu concluir que existem leis $e$ incentivos, principalmente de caráter tributário, que promovem o desenvolvimento das energias renováveis. No entanto, no país ainda é necessário aprofundar estudos que gerem quantificações contínuas do potencial de implementação de fontes não convencionais de energia, de maneira que se melhorem as políticas energéticas, permitindo estabelecer estratégias a longo prazo que envolvam os componentes sociais e ambientais.

Palavras chave: Energias renováveis, Fontes não convencionais de energia, Regulação e incentivos em energias renováveis.

Ortiz, D., Sabogal, J. \& Hurtado. E. (2012) Una revisión a la reglamentación e incentivos de las energías renovables en Colombia. En: Revista de la Facultad de Ciencias Económicas de la Universidad Militar Nueva Granada. rev.fac.cienc.econ, XX (2).

JEL: Q41, Q42, Q56, Q59.
\end{abstract}

\section{Introducción}

Frente al problema ambiental global del cambio climático, las tendencias de crecimiento altamente impactantes por el aumento en el consumo de recursos y energía y la generación de residuos y entropía, surge la oportunidad de desarrollar innovaciones en productos, procesos y servicios que permitan generar bienestar y a la vez reducir la presión sobre el ambiente local, regional y global.

Es en este contexto que surge la generación de tecnologías limpias en el sector de la energía, dinámica que presenta una competencia creciente entre algunos países pioneros europeos, una fuerte actividad de los países del continente Asiático, con China e India como líderes, y las iniciativas de algunos estados y organizaciones de Estados Unidos (Chichilnisky \& Sheeran, 2009).

En contraste a esta tendencia se ven relegadas las regiones en desarrollo como es el caso de África y América Latina, las cuales tienen dificultades en la implementación y desarrollo de este tipo de ener- gías. Esto se puede explicar entre otras razones por: procesos de lock-in tecnológico en tecnologías contaminantes, insipiente desarrollo de procesos de innovación tecnológica y debilidad de las estructuras institucionales en las relaciones sociedad-naturaleza.

En este panorama vale la pena resaltar que los cambios que se den en materia energética en los países desarrollados pueden condicionar el futuro energético global afectando las decisiones y posibilidades de los países en desarrollo como Colombia (Sabogal et al., 2010).

Consecuentemente, y partiendo del potencial que tiene nuestro país en el campo de las energías renovables, dada su ubicación geográfica y la disponibilidad de recursos con que cuenta, se hace necesario analizar la regulación y las políticas existentes, que aseguran una adecuada promoción, producción, desarrollo o adaptación creativa de las energías renovables en el sector energético colombiano y las barreras que se dan a estas alternativas. El aprovechamiento del potencial facilita un acceso más confiable y equitativo a los servicios energéticos, y 
permite una mejora en la estabilidad de los precios, al tiempo que reduce la presión humana de los procesos socio-ambientales.

De otro lado, las directrices de la política energética colombiana referidas a las energías renovables están contenidas en el Plan Energético Nacional; adicionalmente, existe un marco contextual definido por los acuerdos internacionales que ha suscrito el país. La metodología se aplica de esta manera a los programas, planes, leyes y decretos que inciden en la planeación, ejecución y operación de proyectos energéticos.

A partir del análisis de los elementos descritos, la investigación concluye que Colombia debe realizar mayores esfuerzos con el fin de profundizar el conocimiento y clasificación de sus Fuentes No Convencionales de Energía ${ }^{1}$, ésta información permitirá una continua coherencia entre las políticas y estrategias de largo plazo y las condiciones ambientales locales y globales.

De esta manera, el potencial del país dadas sus condiciones biogeoestratégicas, puede ser aprovechado con un incremento y diversificación de su canasta energética y particularmente permitiendo el aprovisionamiento energético en las Zonas No Interconectadas - ZNI del país, mediante la implementación de proyectos que aprovechen nuevas fuentes de energía. Así se podría llegar a que el país alcance un doble resultado positivo: la contribución a la mitigación del problema ambiental del calentamiento global y la participación de los proyectos colombianos en el Mercado de Carbono, accediendo a recursos del Mecanismo de Desarrollo Limpio mediante la venta de Certificados de Reducción de Emisiones.

\section{Contextualización de las energías renovables}

Aunque el fenómeno del cambio climático ha venido siendo objeto de estudio hace ya más de un siglo, sólo en los últimos decenios el incremento de los impactos en los sistemas humanos y ecosistémicos y la consecuente presión social y política, ha generado una mayor conciencia y ha concebido muchos de los cambios que en política internacional se vienen adelantando.

Todo esto, gracias al avance en el consenso por la comunidad científica internacional representada en el Intergovernmental Panel on Climate Change (IPCC) frente a dos hechos fundamentales: en primer lugar el reconocimiento del cambio climático como problema ambiental global y en segundo lugar, la relación establecida entre la actividad humana como causante del fenómeno del cambio climático.

Es así como luego de innumerables negociaciones, en la cumbre de la tierra de 1992 se crea la Convención Marco de las Naciones Unidas para el Cambio Climático (UNFCCC) y con ella nuevamente surge la importancia de abordar el cambio climático como un problema ambiental global. Posteriormente, en 1997 nace el Protocolo de Kioto convirtiéndose en el primer acuerdo climático que establece compromisos cuantificables de mitigación de gases efecto invernadero frente al fenómeno del cambio climático.

A pesar de que en los debates científicos no se ha logrado un consenso total, frente al concepto del cambio climático, éste es definido por la UNFCCC como "un cambio de clima atribuido directa o indirectamente a la actividad humana que altera la composición de la atmósfera mundial y que se suma a la variabilidad natural del clima observada durante periodos de tiempo comparables" (Naciones Unidas, 1992).

Se suma a lo anterior, los resultados que muestran que la acción humana se enfrenta a un fenómeno considerado como irreversible, lo que vuelve aún más complejo su abordaje dado que existe un efecto acumulado de emisiones de gases efecto invernade-

1 El Programa de uso racional y eficiente de energía y fuentes no convencionales (PROURE), en su plan de acción indicativo 20102015, indica que de acuerdo con el decreto 3683 de 2003, articulo 2., se establece que las Fuentes No Convencionales de Energía -FNCE son aquellas fuentes disponibles a nivel mundial que son ambientalmente sostenibles, pero que en el país no son empleadas o son utilizadas de manera marginal y no se comercializan ampliamente. Se consideran fuentes no convencionales de energía, entre otras: la energía solar, energía eólica, energía geotérmica, energía proveniente de fuentes de biomasa, pequeños aprovechamientos hidro-energéticos, energía proveniente de los océanos. 
ro y una permanencia en el tiempo de los impactos del fenómeno ambiental (Chichilnisky \& Sheeran, 2009).

El cambio climático, es una de las expresiones frecuentes en los actuales debates en el marco de la dicotomía entre crecimiento económico y ambiente. El centro de la estrategia de los países en términos de desarrollo económico, depende en buena medida del consumo energético de combustibles fósiles con la consecuente emisión de gases efecto invernadero $y$ este es un ciclo que se repite con un incremento continuo de impactos ambientales en el sistema climático global (Sabogal \& Moreno, 2011).

Los países industrializados, principales causantes del cambio climático, han reconocido su responsabilidad pero no están dispuestos a una reducción en su consumo energético, por su parte los países en desarrollo se niegan a aceptar una limitación a sus consumos energéticos que les permitan el desarrollo de sus economías.

Dada esta dicotomía se busca la reducción de emisiones en la atmósfera sin limitar las posibilidades de crecimiento económico. En ese contexto, Baeza (2007, 2), afirma que "la generación eléctrica debe garantizar el suministro, asegurar unos costes energéticos competitivos para la economía productiva y garantizar la sostenibilidad medioambiental", de manera que los modelos del mercado energético, las fuentes de energías y tecnologías y la gestión de la demanda energética, se convierten en herramientas para lograr este objetivo.

En otras palabras, se está en mora del diseño de una solución que incluya las limitaciones del consumo de energía, buscando alternativas que garanticen que tales cambios no afecten negativamente la demanda energética.

Una búsqueda de alternativas energéticas se justifica debido a que el elevado consumo energético que se viene registrando en las últimas décadas, trae consigo problemas de sostenibilidad: ambiental, social y económica. A resaltar en esta discusión se encuentra, la relación entre el aumento en el ritmo de consumo energético y el stock de combustibles fósiles; los problemas ambientales globales relacionados con el aumento en la emisión de Gases Efecto Invernadero; y los problemas sociales y políticos debidos a las tensiones entre países productores y consumidores del petróleo (González, 2009).

\section{Nuevos desarrollos tecnológicos}

Pese a algunos de los problemas tanto de sostenibilidad como ambientales relacionados con el proceso de utilización de la energía, se reconoce que no se puede obviar que el consumo energético permite satisfacer muchas de las necesidades básicas, entre ellas: el suministro de agua potable, iluminación, capacidad de producir, transportar y procesar alimentos, la movilidad y el acceso a la información, entre otras (Pérez, 2002).

De acuerdo a esto, el centro del debate está en que para satisfacer estas necesidades se utiliza la energía fósil, la cual tiene impactos cada vez mayores en el sistema climático global. De manera que estamos ante una situación que no es fácil de re-direccionar, teniendo presente que los combustibles fósiles tienen una participación central en los sistemas de producción-consumo posteriores a la revolución industrial (IEA, 2008).

Es precisamente ante los efectos negativos de la utilización de combustibles fósiles y la emisión de gases efecto invernadero, que surgen avances tecnológicos en favor de energías más limpias. Baños et al. (2011) señala a este respecto, que las energías limpias pretenden reducir la dependencia de los combustibles fósiles, disminuir la emisión de gases efecto invernadero y evitar problemas de seguridad ocasionados por la energía atómica, entre otros.

Es así como han surgido iniciativas tecnológicas y un rápido crecimiento del mercado de las energías renovables, aunque es un aumento que deja ciertas dudas teniendo como referente el incremento registrado en el uso del carbón y del petróleo.

Estas energías limpias o energías renovables, definidas como "aquellas cuyo flujo es repuesto, a partir de fuentes naturales, al mismo ritmo con que se consumen y la característica de estas fuentes es que es- 
tán dispersas por todo el planeta" (González, 2009, VI), son una de las formas en que el ser humano ve la posibilidad de salir de un bloqueo tecnológico de las energías de origen fósil, la utilización de otras formas con menor impacto en los sistemas ambientales y humanos y una mayor posibilidad de sostenibilidad en el tiempo.

En una revisión del panorama mundial respecto a este tipo de energías alternativas o renovables, se ha encontrado que el mercado líder en términos de tecnología energética es el europeo, el cual comenzó hace varias décadas con monopolios regionales y nacionales con alto apoyo gubernamental. Esta evolución viene acompañada de un proceso de cambio que ha consistido en que las grandes empresas separaran sus actividades económicas y perfeccionan la búsqueda de conexiones o nuevas sinergias en el mercado (Baeza, 2007).

El continente asiático con China e India como líderes, comienza a posicionarse como pionero en la generación hidroeléctrica de energía. Por su parte los países en desarrollo como los de América del Sur y África, a pesar de que tienen gran potencial, tienen dificultades con el desarrollo de este tipo de energías, asociadas a influencias directas que ejercen el crecimiento económico y el aumento de la demanda energética (Flamos, 2010).

De otro lado, Infante (2007) afirma que las estadísticas evidencian que Estados Unidos es el país de América más dependiente de las fuentes tradicionales, demandando casi el $86 \%$ del total de energía, del cual el $40 \%$ proviene del petróleo. A partir de allí, las cifras registradas en relación con el uso actual de energías fósiles generan una gran preocupación por la poca injerencia de las fuentes renovables de energía y se comienza a gestar un cambio importante en cuanto a producción y consumo.

Los cambios registrados en las potencias mundiales como la Unión Europea, China y Estados Unidos direccionan en gran medida el futuro energético global, pues lo que puedan modificar en sus países generará un efecto significativo en el panorama mundial ener- gético, ampliando las oportunidades para países en vía de desarrollo como Colombia.

Por otro lado, países de la Unión Europea, Japón, Australia y Corea del Sur, son otros de los que se suman a Estados Unidos en la creación de políticas radicales que promueven cambios importantes hacia la implementación de energías renovables, encaminadas a disminuir la dependencia del petróleo y sus derivados. En Europa, específicamente en Alemania, es relevante esta necesidad dados los altos niveles de importación de petróleo (Infante, 2007).

En el caso de Latinoamérica, la energía renovable que más predomina es la hidroeléctrica con una participación de $62 \%$ de la oferta total en este tipo de energía, le siguen los biocombustibles con el $36 \%$ y en menor medida la energía eólica y solar. Adicionalmente, está comprobado que los países latinoamericanos cuentan con recursos favorables para el desarrollo de energías limpias, pero se encuentra muy poca voluntad política que incentive la inversión y promueva el desarrollo de proyectos que beneficien la aplicación de este tipo de energías (Canseco, 2010).

Sin embargo, es importante mencionar que en algunos países la reglamentación o normatividad antes de actuar como una herramienta que promueva el desarrollo e implementación de este tipo de energías alternativas, se convierte en algo negativo para algunas empresas privadas, dado que la mayoría están definidas para empresas del sector público, haciendo inequitativa la competencia en el sector.

\section{Reglamentación e incentivos de las energías renovables en Colombia}

Luego de analizar el panorama global de las energías renovables y de revisar el avance de las potencias económicas frente al tema, es necesario estudiar el avance local en la implementación de este tipo de energías. Para tal efecto se debe empezar por destacar el potencial que tiene Colombia en el campo de las energías renovables, gracias tanto a su ubicación geográfica como a los recursos con que cuenta. A continuación se presenta el panorama colombiano respecto al tema. 


\subsection{Las energías renovables: el caso colombiano}

Gracias al estudio de las energías limpias se han desarrollado importantes avances en los productos derivados de ellas. Al respecto, el Plan Energético Nacional 2006-2025 proyecta un panorama favorable tanto en el consumo como en la capacidad de producción de nuevas tecnologías, cita como ejemplo el caso del sector transporte con vehículos impulsados por "Celdas de Combustible" que aumentarían la eficiencia de los motores de combustión en dos y hasta tres veces más.

Adicionalmente, plantea que uno de los programas que se pretende incentivar está orientado a la producción de agrocombustibles, mediante el incremento de productos como el alcohol a partir de materias primas diferentes a las convencionales, dentro de las que se encuentran: maíz, yuca, sorgo, remolacha o cualquier otro tipo de biomasa de azúcar (UPME, 2007).

El horizonte del biocombustible se enfoca a la producción a partir del aceite de palma hasta sustituir un $5 \%$ de la demanda de ACPM (UPME 2007). Vale la pena aclarar que, en Colombia se fomenta la producción y uso de los agrocombustibles a pesar del gran debate existente entre el uso de energías renovables y la seguridad alimentaria, al destinar muchos de los cultivos de alimentos para la producción de energía, los impactos ambientales de los monocultivos y los conflictos por posesión y utilización de tierras.

\subsubsection{Energía solar}

Algunos de los estudios que se adelantan en materia energética en el país son desarrollados por el Instituto de Hidrología, Meteorología y Estudios Ambientales (IDEAM) que en conjunto con la Unidad de Planeación Minero Energética (UPME) desarrollan desde 2004 un documento de referencia en el país que contiene 13 mapas específicos en "Energía Solar", uno para cada mes del año y uno adicional que compila el promedio anual; este documento se conoce como "Atlas de Radiación Solar de Colombia", definido como un compendio de mapas que evidencian el valor promedio diario de radiación solar global, brillo y radiación ultravioleta por metro cuadrado.
Este instrumento permite: i) Identificar las zonas estratégicas para el desarrollo de este tipo de energía, para lograr con éxito la implementación de tecnologías como sistemas fotovoltaicos o térmicos que transformen tal potencial en electricidad, tecnologías encaminadas a la destilación solar para la separación de contaminantes, tecnologías que logren la climatización de edificios e incluso como fuente directa para la producción de biomasa. ii) Determinar las zonas más favorables dentro del territorio colombiano para el desarrollo de este tipo de energía, dentro de las que el informe destaca La Guajira y la Orinoquia. iii) Establecer el documento como un referente técnico y científico para las áreas de: biología, agronomía, ecología, ingeniería energética, y medicina, entre otras (IDEAM \& UPME, 2005).

En el Atlas de Radiación Solar de Colombia, se encuentra el mapa de Radiación Solar Global para 2005, que destaca las zonas más favorables para el desarrollo de proyectos que involucren Energía Solar, dentro de las cuales se encuentran los departamentos de: La Guajira, Atlántico, Magdalena, Sucre; la zona norte de los departamentos de Bolívar, Cesar y Córdoba; y el nororiente de los departamentos de Arauca y Vichada. Al comparar este documento con otros similares que anteriormente había desarrollado el IDEAM en este se evidencia "la influencia de las cordilleras y de las zonas planas, encontrándose que la distribución de los rangos de valores de la radiación solar refleja la existencia de las cordilleras" (IDEAM \& UPME, 2005).

Por otra parte, Rodríguez (2009) comenta que a partir de una revisión de la energía solar en Colombia, ha encontrado que las primeras aplicaciones se introdujeron a mediados del siglo anterior, con la instalación de calentadores solares en Santa Marta. Más adelante por la década de los setenta, la crisis del petróleo incentivo nuevamente la energía solar $y$ con apoyo de universidades y fundaciones se instalaron calentadores en centros de servicios comunitarios como hospitales, sin embargo, tan solo hasta la década de los ochenta surgieron algunos casos de implementación de calentadores en urbanizaciones de Medellín y Bogotá. 


\subsubsection{Energía hidráulica}

El Plan Energético Nacional 2006-2025, contempla que la energía hidráulica es la más desarrollada en Colombia, representando en promedio el $75 \%$ de la generación de electricidad. Lo anterior, pese a los efectos que este tipo de energía ocasiona en el ambiente, como desplazamientos de biodiversidad generados por la inundación de grandes extensiones de tierras, donde se obliga a muchas de las especies a desplazarse o eventualmente a su extinción. Esto también ha sido la causa del desplazamiento o migración de muchos asentamientos humanos aledaños a las zonas de intervención.

La implementación de Pequeñas Centrales Hidroeléctricas, hace que disminuyan en gran medida estos efectos secundarios para el hábitat. Muchas de estas centrales son clasificadas como proyectos MDL; en el documento de Canseco $(2010,11)$ se encuentra un proyecto de este tipo denominado "Desplazamiento de la Electricidad de la Red Eléctrica Nacional por la Autogeneración de Energía Renovable en la Planta de Tratamiento de Aguas Residuales Cañaveralejo", el cual tuvo como objetivo generar electricidad desde una planta de tratamiento de aguas residuales hasta satisfacer el consumo de la misma central (UPME 2007).

Por lo anterior, el IDEAM presentó el Estudio Nacional del Agua en 2010, este documento trae una compilación del estudio de aguas superficiales, aguas subterráneas y la estimación de la demanda de agua, entre muchos otros estudios. La construcción de este documento se soporta en información suministrada por el Sistema de Información Ambiental (SIA), junto con la red de referencia del IDEAM, compuesta por estaciones hidrológicas y meteorológicas dispuestas a lo largo del país que brindan información real del estado de la atmosfera e hidrosfera. El monitoreo de estas estaciones apoyado por imágenes satelitales permiten evaluar diariamente el comportamiento de las condiciones hidrológicas, meteorológicas y ambientales de todo el país, con el fin de emitir alertas tempranas y evitar desastres ambientales.

\subsubsection{Energía eólica}

De acuerdo a Barba et al. (2009), en el capítulo tres "Mitigación" de la segunda comunicación nacional ante la Convención Marco de las Naciones Unidas sobre Cambio Climático, en el "Atlas de Viento y Energía Eólica en Colombia" se presenta la distribución espacial del viento en superficie mediante una colección de mapas que evidencian el promedio anual y mensual de los vientos, algunos estadísticos importantes como la desviación estándar y el rango para cada mes y específicamente en el caso de la energía eólica la densidad mensual y anual que permite identificar no solo zonas del país sino épocas del año favorables para el desarrollo de este tipo de energía.

En los resultados de este Atlas de Viento, se destacan 16 lugares de Colombia aptos para el desarrollo e implantación de tecnologías a favor de este tipo de energía, tres de estos lugares presentan vientos "persistentes y superiores a $5 \mathrm{~m} / \mathrm{s}$ durante todo el año: Galerazamba en el Departamento de Bolívar, Gachaneca en Boyacá y la isla de San Andrés en el mar Caribe colombiano"; otros tres lugares se destacan porque las "velocidades son persistentes pero en el rango entre los 4 y $5 \mathrm{~m} / \mathrm{s}$ : La Legiosa en el Huila, Isla de Providencia en el Mar Caribe y Riohacha en La Guajira"; los restantes diez lugares a pesar de que no presentan una continuidad en velocidad, determinan épocas especiales del año como son: "Villacarmen en Boyacá, Obonuco en Nariño, Cúcuta y Ábrego en Norte de Santander, Urrao en Antioquia, Soledad en Atlántico, Santa Marta en Magdalena, Bucaramanga en Santander, Anchique en Tolima y Bogotá en Cundinamarca" (IDEAM \& UPME, 2006, 15).

Frente a este mismo tema, el Plan Energético Nacional 2006-2025 destaca el proyecto Jepirachi, como un proyecto innovador en el país, con una capacidad instalada de 19,5 MW, y cuya generación de energía se considera limpia porque no produce efectos secundarios y al mismo tiempo ayuda a reducir los gases efecto invernadero sin involucrar combustión de ningún tipo. Sin embargo, a pesar de estas ventajas, se debe tener en cuenta que la intermitencia de los vientos puede generar bajas tasas de producción (UPME, 2007). 
Jepirachi fue llevado a cabo por la suma de varios factores y agentes entre los cuales se destacan: la apuesta realizada por las Empresas Públicas de Medellín, quien asumió el riesgo financiero para la implementación del mismo, la velocidad de los vientos en la Guajira, las condiciones del puerto, las facilidades de transporte terrestre y una zona aduanera especial, entre otras (UPME, 2007).

El mapa de la velocidad media del viento en superficie, extraído del Atlas de Viento y Energía Eólica de Colombia, evidencia que la medición promedio de vientos durante todo el año en la península de la Guajira oscila entre 5 y $11 \mathrm{~m} / \mathrm{s}$; en el resto del país durante los meses de abril, octubre y diciembre los vientos se debilitan, pero en los demás meses se alcanza intensidades de hasta $4 \mathrm{~m} / \mathrm{s}$, en el litoral central de Bolívar y Atlántico, límites entre Boyacá y Cundinamarca, Piedemonte Llanero de Meta y Casanare (IDEAM \& UPME, 2006).

\subsubsection{Energía por biomasa}

Otro Atlas que es importante mencionar es el "Atlas del Potencial Energético de la Biomasa Residual en Colombia", en este documento desarrollado por la UPME, el IDEAM, El Departamento Administrativo de Ciencia, Tecnología e Innovación -COLCIENCIAS-, y la Universidad Industrial de Santander -UIS, se valora la oferta energética de biomasa contenida en algunas especies representativas de los sectores agrícola, pecuario y residuos orgánicos urbanos, mediante mapas que muestran la cantidad de biomasa residual producida en nuestro país, las áreas cultivadas, la población pecuaria, y el volumen de residuos sólidos orgánicos urbanos (UPME et al., 2009).

El documento anterior arroja resultados importantes dentro de los que cabe destacar: que el $44,77 \%$ de la superficie continental colombiana está destinada a la actividad agropecuaria, además se evidencia una gran variedad de hábitats acuáticos con infinidad de biodiversidad que ayudan en la producción de biomasa, entre ellos las algas consideradas como un posible insumo para la producción de biodiesel.

Consecuentemente, existe registro de que en Colombia el sector pecuario cuenta con el $76 \%$ del área to- tal destinada a actividades agropecuarias, esta cifra se compone de un $40 \%$ de actividades bovinas, un $40 \%$ de actividades avícolas un $10 \%$ de actividades porcícola y el restante $10 \%$ corresponde a actividades caprinas, ovinas, piscícola y equina principalmente. Todos los datos anteriores se traducen en enormes cantidades de estiércol, y esta a su vez es uno de los insumos principales para la obtención de energía de biomasa residual (UPME et al., 2009).

\subsubsection{Energía geotérmica}

La UPME en el año 2003, presentó un documento para la utilización de la energía geotérmica en Colombia, en el que se evidencia el desarrollo de las investigaciones que en esta materia se han llevado a cabo en el país, partiendo de 1997 cuando fue perforado el primer pozo Nereidas en el macizo Volcánico del Ruiz, desde entonces se realizó un estudio que generó como resultado el mapa geotérmico de Colombia, el cual contiene los yacimientos termales y la temperatura de los pozos existentes.

En dicho documento se plantean además, algunos usos adicionales a la generación de electricidad como por ejemplo, calefacción, lavado y secado de lana, procesamiento de alimentos, fermentación, producción de ácido sulfúrico, industria papelera, manufactura de cemento, entre otras. Adicionalmente, se determinan 4 zonas importantes de estudio: El Azufral, el proyecto binacional Chiles, Cerro Negro y Tufiño, el de Paipa y el Proyecto del Nevado del Ruiz.

La implementación de la energía Geotérmica en Colombia, apenas empieza a desarrollarse, gracias al apoyo del Fondo para el Medio Ambiente Mundial, administrado por el Banco Interamericano de Desarrollo, que lleva más de tres decenios promoviendo el desarrollo de este tipo de energía renovable en Latinoamérica. Las entidades colombianas que se encuentran al frente del desarrollo del proyecto son: $\mathrm{El}$ Ministerio de Minas y Energía y la empresa ISAGEN que se encargará de la implementación.

\subsubsection{Energía oceánica}

En el caso de la energía oceánica, el potencial de generación a lo largo de la costa colombiana esta 
dado por el uso de corrientes inducidas por mareas, técnica que requiere de tecnologías mucho más avanzadas de las actuales, para el aprovechamiento del recuso energético. En los estudios de este tipo de energías en la costa pacífica colombiana se han podido establecer varias bahías con amplios potenciales de generación eléctrica por corrientes, es el caso de: La Bahía Punta Catripe, Bahía Delta Chavica, Bahía Bocana Raposo, Bahía Boca Naya y Bahía Bocana Armerías (Polo et al., 2008).

Polo et al. (2008, 100), se refiere a la energía de las olas, enunciando las corrientes que afectan las costas colombianas: "en el Atlántico por la corriente del Nordeste del Brasil, que posteriormente alimenta la corriente del Golfo, y en el Pacífico por la corriente de Humbolt, que se mueve por la costa occidental de Suramérica desde el Ártico y es una corriente fría".

\subsection{Reglamentación colombiana}

Es necesario tener presente que en Colombia, la política energética se encuentra definida en el Plan Energético Nacional, adicionalmente el Estado colombiano expide leyes que le permiten mantener un control de las estrategias a desarrollar en el sector, como: planes, decisiones y regulaciones. También cabe mencionar que Colombia es firmante y parte activa de acuerdos internacionales ambientales (AMUMAS).

El Programa de Uso Racional y Eficiente de Energía y Fuentes No Convencionales (PROURE), en su plan de acción indicativo 2010-2015, (UPME, 2010a, presenta las metas para las Fuentes No Convencionales de Energía (FNCE), donde propone para el año 2015 alcanzar un aumento en las FNCE del 5\% de la canasta energética total, de los cuales el $4 \%$ correspondería al uso de biomasa y biocombustibles y el 1\% restante a energía eléctrica, estimaciones establecidas desde los datos del estudio así:

"En 2008 la participación de energéticos renovables en el consumo final de energía fue de 6,5\% en biocombustibles, $10,4 \%$ en bagazo y $20,6 \%$ en la utili- zación de leña, con base en las estimaciones preliminares de inventarios y potenciales de los recursos energéticos renovables y su participación en el mix nacional". (UPME, 2010a, 9).

El mismo documento sugiere que para el año 2020 se alcanza el 6,5\% de capacidad total instalada en concordancia con la reducción de los costos de tecnologías, estrategias, estudios y planes desarrollados por los generadores de energía eléctrica. Adicionalmente contempla que la capacidad instalada para las Zonas No Interconectadas a 2015 se debe incrementar a $20 \%$ (en comparación al $8 \%$ que abarcaba en 2008), el $12 \%$ pendiente por crecer debe estar desarrollado por sectores de energía eólica, biomasa, pequeñas centrales hidroeléctricas y energía solar. A 2020 la meta se incrementa a un 30\% (UPME, 2010a).

El Plan Energético Nacional 2006-2025, se refiere a este tema planteando algunos factores que resultan decisivos para el uso de sistemas de generación y energización dentro de los que se encuentran: "los subsidios a los combustibles, costos de infraestructura del transporte y almacenamiento de combustibles sufragados por el público, disponibilidad de financiación para proyectos de bajo costo inicial, ausencia de cobros o penalidades por impactos ambientales $y$ posiblemente la resistencia al cambio" (UPME, 2007, 206).

En el mismo sentido, y con el objetivo de reglamentar el desarrollo, uso e implementación de las energías renovables en el país, además de estimular el cumplimiento de las metas propuestas y brindar incentivos para los desarrolladores e implementadores de estas nuevas energías, existe un marco normativo que se detalla a continuación en la Tabla 1.

\section{Conclusiones}

El potencial de Colombia dada su riqueza en recursos energéticos la ubica en una situación privilegiada frente a otros países de la región; esto se traduce en una ventaja que le permite aumentar y diversificar su canasta energética, pese a ello, se hace necesario un aprovechamiento de este potencial que se vea 
Tabla 1. Marco Normativo Relacionado con Energía Renovables en Colombia

\begin{tabular}{|c|c|}
\hline Normatividad & Descripción \\
\hline Ley 51 de 1989 & $\begin{array}{l}\text { En esta ley se crea la Comisión Nacional de Energía que es la encargada de determinar funciones de } \\
\text { planeación energética, además de "efectuar, contratar o promover la realización de estudios para estable- } \\
\text { cer la conveniencia económica y social del desarrollo de fuentes y usos energéticos no convencionales y } \\
\text { adoptar la política respectiva" y en relación con el subsector de energía eléctrica, "aprobar los programas } \\
\text { de generación eléctrica no convencional y coordinar los programas de generación eléctrica en áreas no } \\
\text { interconectadas" (UPME, 2010b, 2-4). }\end{array}$ \\
\hline Decreto 2119 de 1992 & $\begin{array}{l}\text { Mediante este decreto se reestructuro el Instituto de Asuntos Nucleares -IAN-, y cambio de nombre a Ins- } \\
\text { tituto de Asuntos Nucleares y Energías Alternativas -INEA- donde se suma a sus funciones el de fomentar } \\
\text { el uso racional de la energía, un aparte textual del decreto afirma "Igualmente el INEA deberá elaborar } \\
\text { programas científico y tecnológicos para la intensificación del uso de las fuentes alternas de energía". } \\
\text { Con el fin de dar cumplimiento a su labor, en el artículo } 44 \text { se crea el comité de Uso Racional de Energía } \\
\text {-URE- (UPME } 2010 b, 2-5) \text {. }\end{array}$ \\
\hline Ley 142 de 1994 & $\begin{array}{l}\text { La ley } 142 \text { resulta ser la pionera en la regulación de los servicios públicos domiciliarios en el país, in- } \\
\text { centivando una fuente no convencional de energía como lo es el gas natural. Esta ley le impone a la } \\
\text { Comisión de Regulación de Energía y Gas, funciones para la regulación en cuanto a autogeneración y } \\
\text { cogeneración de energía. } \\
\text { Adicionalmente, en esta ley también se definen políticas para la gestión integral de residuos sólidos y } \\
\text { peligrosos con el objetivo de minimizar los impactos al hombre y al medio ambiente. }\end{array}$ \\
\hline Ley 143 de 1994 & $\begin{array}{l}\text { La ley } 143 \text { es conocida como la "Ley Eléctrica", en esta ley se le atribuye a la UPME la función de elaborar } \\
\text { y actualizar el Plan Energético Nacional -PEN-, el cual debe brindar los lineamientos para el desarrollo } \\
\text { del sector energético en el país. Esta ley se constituyó en la gran impulsadora de proyectos hasta finales } \\
\text { de la década del noventa cuando la recesión económica golpeó fuertemente el sector y condujo al de- } \\
\text { sarrollo de proyectos en los sectores de mayor confianza en el país como el hídrico y el carbón mineral. } \\
\text { Consecuentemente en esta Ley, se designaron las actividades de generación, interconexión, transmi- } \\
\text { sión, distribución y comercialización de la electricidad, al mismo tiempo, dispuso que el Estado debía } \\
\text { ser el encargado de "asegurar la adecuada incorporación de los aspectos ambientales en la planeación } \\
\text { y gestión de las actividades del sector" y "Abastecer la demanda de electricidad bajo criterios económi- } \\
\text { cos y de viabilidad financiera, asegurando su cubrimiento en un marco de uso racional y eficiente de los } \\
\text { diferentes recursos energéticos del país" (UPME, 2010b, 2-5). }\end{array}$ \\
\hline Decreto 1682 de 1997 & $\begin{array}{l}\text { Mediante el cual se suprime el INEA y se asignan sus funciones a la UPME, de esta manera se concentró } \\
\text { en la UPME la planeación energética de todos los recursos incluyendo las FNCE, con el fin de estructurar } \\
\text { planes integrales que articulen diferentes frentes y que tengan en cuenta la Oferta y la Demanda (UPME, } \\
\text { 2010b). }\end{array}$ \\
\hline Ley 697 de 2001 & $\begin{array}{l}\text { La ley } 697 \text { es la que promueve la utilización de energías alternativas, además pone en cabeza del Minis- } \\
\text { terio de Minas y Energía la responsabilidad de promover y adoptar programas para este tipo de energías. } \\
\text { Mediante esta ley se declara el Uso Racional y Eficiente de la Energía -URE-, y sus objetivos se definen } \\
\text { en el Artículo 1, como: "Asegurar el abastecimiento energético pleno y oportuno, la competitividad de la } \\
\text { economía colombiana, la protección al consumidor y la promoción del uso de energías no convenciona- } \\
\text { les de manera sostenible con el medio ambiente y los recursos naturales". }\end{array}$ \\
\hline Ley 788 de 2002 & $\begin{array}{l}\text { Ley mediante la cual se modifica el estatuto tributario para "incentivar la compra e implementación de } \\
\text { equipos y tecnologías que demuestren impacto en la mitigación del cambio climático", en el artículo } 18 \\
\text { de dicha ley se contempla "la exención de renta por } 15 \text { años, a la venta de energía producida a partir de } \\
\text { fuentes renovables como la eólica, biomasa o residuos agrícolas". } \\
\text { Con la implementación de este beneficio se espera favorecer a las empresas generadoras, siempre que } \\
\text { estas vendan los certificados de reducción de GEl y destinen como mínimo el } 50 \% \text { de las ganancias a } \\
\text { nueva inversión en las zonas en que se ejerce su actividad. } \\
\text { En el artículo } 95 \text { de la misma ley, también se encuentran exenciones a los importadores de maquinarias } \\
\text { que introduzcan al país equipos destinados a proyectos que contribuyan con la reducción de GEl, estos } \\
\text { estarán exentos del pago del impuesto de valor agregado -IVA- (Barba et al., 2009). }\end{array}$ \\
\hline
\end{tabular}

2 Fuente: Elaboración de los autores a partir de documentos en las referencias bibliográficas. 


\begin{tabular}{|l|l|}
\hline Decreto $\mathbf{3 6 8 3}$ de $\mathbf{2 0 0 3}$ & $\begin{array}{l}\text { Este decreto reglamentario crea la Comisión Intersectorial de Uso Racional de Energía -CIURE-, la cual } \\
\text { es presidida por el Ministerio de Minas y Energía, e integrada por el Ministro de Comercio, Industria y } \\
\text { Turismo, Ministro de Ambiente, Vivienda y Desarrollo Territorial, el director del DNP, el director ejecutivo de } \\
\text { la CREG, el director de COLCIENCIAS y el director del IPSE. El objetivo primordial de esta comisión es } \\
\text { el de servir como articulador, asesor o consultor de las entidades estatales para facilitar la ejecución de } \\
\text { políticas específicas para el sector (UPME, 2010b). }\end{array}$ \\
\hline $\begin{array}{l}\text { Resolución } \mathbf{0 0 4 7} \text { de } \\
\text { Abril 14 de } \mathbf{2 0 0 3}\end{array}$ & $\begin{array}{l}\text { Dicha resolución estipula la calidad del Alcohol Carburante (etanol anhidro), y las gasolinas oxigenadas, } \\
\text { el uso de los aditivos en las gasolinas colombianas y la calidad del combustible diesel (ACPM). }\end{array}$ \\
\hline $\begin{array}{l}\text { Resolución 18-919 de de } \mathbf{1 0} \text { de } \\
\mathbf{2 0 1 0}\end{array}$ & $\begin{array}{l}\text { Resolución que en su artículo primero "Adopta el plan de acción indicativo 2010-2015 para desarrollar } \\
\text { el programa de Uso Racional y Eficiente de la Energía y demás formas de energía no convencionales } \\
\text { PROURE". } \\
\text { En su artículo 2 se define el objetivo del plan como aquel que contribuya a "asegurar el abastecimiento } \\
\text { energético pleno y oportuno, la competitividad de la economía colombiana, la protección al consumidor } \\
\text { y la promoción del uso de energías no convencionales de manera sostenible con el ambiente y los re- } \\
\text { cursos naturales". }\end{array}$ \\
\hline
\end{tabular}

reflejado en la implementación efectiva de proyectos de energía renovable como una alternativa en concordancia con los requerimientos de sostenibilidad ambiental, social y económica para el desarrollo energético del país.

Sin embargo, reconocer el amplio potencial con que cuenta el país o las carencias al implementar proyectos que promuevan la investigación, desarrollo y aplicación de proyectos de energías renovables no es suficiente; debe promoverse la tendencia de sustitución de combustibles fósiles por fuentes alternativas de energía, es el momento oportuno para que se tomen acciones en cuanto al desarrollo e implementación de proyectos en energías renovables.

Organizaciones públicas y privadas han mostrado su interés en la realización de proyectos con energías renovables. Cabe destacar el caso de las Empresas Públicas de Medellín, que asumió el riesgo de la implementación del primer parque eólico en el norte de Colombia. Sin embargo, se requiere un mayor apoyo gubernamental con recursos financieros y acompañamiento.

De otro lado, debe reconocerse que la energía hidroeléctrica tiene tradición y amplia participación en la canasta energética nacional, pero, se requiere al respecto una evaluación de los impactos ambientales de su construcción y funcionamiento, la realización de pequeñas hidroeléctricas para zonas con intereses particulares y sabiendo los impactos que sobre la oferta tienen los fenómenos cada vez más intensos y frecuentes del niño y la niña.
Al mismo tiempo, las energías eólica y geotérmica, requieren un marco jurídico que haga posible su desarrollo, papel de las entidades reguladoras, y una política clara de parte del Estado colombiano para incentivar la innovación y desarrollo de estas energías; así mismo, la energía mareomotriz, oceánica y de biomasa involucran entidades que requieren una coordinación en el orden nacional y regional.

A la par de la necesidad de implementar los proyectos, también se hace necesario el fortalecimiento de las entidades nacionales relacionadas y de instituciones y mecanismos que fomenten las investigaciones, de tal forma que se incremente el conocimiento y cuantificación de los recursos energéticos.

De esta manera se puede generar información para fortalecer las instituciones, las políticas, las leyes, las normas y los decretos. Es el caso del IDEAM y de la UPME, instituciones dedicadas a la cuantificación de este tipo de energías, que podrían asumir el papel de gestoras de cambio para el desarrollo e innovación de energías como la hidráulica y otras que hemos identificado como de alto potencial.

A pesar de que en Colombia ya se ha dado un primer paso hacia el establecimiento de políticas en energías renovables, se hace necesaria la coordinación con las estrategias de los principales sectores de la economía, para de esta manera ampliar el cubrimiento de las mismas y hacer más efectiva su implementación. En este sentido las leyes y decretos (generados antes de 2001), abrieron el 
camino y plantearon lineamientos de política en el país.

Sin embargo, los bajos índices de desarrollo e implementación hacen más evidente la necesidad de generar incentivos en el sector energético. El caso contrario se presenta en el sector de los biocombustibles, donde se observan indicadores positivos que demuestran que los incentivos aplicados al sector han surtido el efecto esperado, pero en este caso particular un balance ambiental, social y económico permitiría redefinir las políticas e instrumentos para aumentar el beneficio general.

La importancia de la implementación de buenas y mejores políticas en el campo de las energías renovables permitiría generar los incentivos necesarios para que los proyectos favorezcan a las comunidades alejadas y pertenecientes a las zonas no interconectadas $y$ a los proyectos que aporten en los aspectos ambientales, sociales y económicos.

\section{Referencias}

Baeza, R. (2007). "Horizonte a Largo Plazo en la Generación Eléctrica". En: Revista de Economía Industrial, Ministerio de Ciencia y Tecnología Español, 364: 87-102.

Baños, R. Manzano-Agugliaro, F. Montoya, F, Gil, C, Alcayde, A. \& Gómez, J. (2011). "Optimization Methods Applied to Renewable and Sustainable Energy: A Review". En: Renewable and Sustainable Energy Reviews. Expert Systems with Applications, 15: 1753-1766.

Barba, D., Garavito, S., López, S., Nieves, H., Olarte, C., Ortega, S. \& Santos, L. (2009). Mitigación. En: Riveros, V., - Scripto Ltda. (Eds.). Segunda Comunicación Nacional ante la CMNUCC. Bogotá, Colombia.

Canseco, M. (2010). Energías Renovables en América Latina. En: Fundación Ciudadanía y Valores. Madrid-España: 1-12.

Chichilnisky, G. \& Sheeran, K. (2009). Saving Kyoto. London: New Holland Publishers (UK).

Flamos, A. (2010). "The Clean Development Mechanism - Catalyst for Wide Spread Deployment of Renewable Energy Technologies? or Misnomer?". En: Environment, Development and Sustainability. SpringerLink, 12 (1): 89-102.

González, J. (2009). Aprovechamiento térmico de la energía solar. En: Reverté S.A. (Eds.). Energías Renovables. (60-93). Loreto, Barcelona, España.

International Energy Agency - IEA (2008). World Energy Outlook. Paris: IEA.

Infante, A. (2007). "Perspectivas de la Situación Energética Mundial. Las oportunidades para Colombia". En: Revista de Ingeniería, Universidad de los Andes, 25: 1-28.
Instituto de Hidrología, Meteorología y Estudios Ambientales (IDEAM) \& Unidad de Planeación Minero Energética (UPME) (2005). Atlas de radiación solar de Colombia. Bogotá. Instituto de meteorología, hidrología y estudios ambientales; Unidad de planeación minero energética (eds.) Bogotá, Colombia.

Instituto de Hidrología, Meteorología y Estudios Ambientales (IDEAM) \& Unidad de Planeación Minero Energética (UPME) (2006). Atlas de viento y energía eólica de Colombia. Bogotá. Instituto de meteorología, hidrología y estudios ambientales; Unidad de planeación minero energética (eds.) Bogotá, Colombia.

Instituto de Hidrología, Meteorología y Estudios Ambientales (IDEAM) (2010). Estudio Nacional del Agua. Bogotá. Instituto de meteorología, hidrología y estudios ambientales (eds.) Bogotá, Colombia.

Ley 142 de 1994. Por la cual se establece el régimen de los servicios públicos domiciliarios y se dictan otras disposiciones. D.O. No. 41.433 .

Ley 697 de 2001. Mediante la cual se fomenta el uso racional y eficiente de la energía, se promueve la utilización de energías alternativas y se dictan otras disposiciones. D.O. No. 44.573.

Naciones Unidas. (1992). Convención Marco de las Naciones Unidas sobre el Cambio Climático.

Pérez, J. (2002). "Energía y Desarrollo Sostenible". Discurso leído en el acto de su recepción pública en la Real Academia de Ingeniería. Disponible en: http://www.oei.es/decada/portadas/ MedAmbPDF4.pdf

Polo, J., Rodríguez, J. \& Sarmiento, A. (2008). "Potencial de Generación de Energía a lo Largo de la Costa Colombiana Mediante el Uso de Corrientes Inducidas por Mareas". En: Revista de Ingeniería. Universidad de los Andes, 28: 99-105.

Rodríguez, H. (2009). "Desarrollo de la energía solar en Colombia y sus perspectivas". En: Revista de Ingeniería. Universidad de los Andes, 28: 83-89.

Resolución 0047 del 14 de abril de 2003. Ministerio de Ambiente, Vivienda y Desarrollo Territorial Ministerio de Minas y Energía. Por la cual se modifica parcialmente la Resolución 898 del 23 de agosto de 1995, que regula los criterios ambientales de calidad de los combustibles líquidos y sólidos utilizados en hornos y calderas de uso comercial e industrial y en motores de combustión interna de vehículos automotores.

Resolución 18-919 de 2010. Ministerio de Minas y Energía. Por el cual se adopta el plan de acción indicativo 2010-2015 para desarrollar el Programa de uso racional y Eficiente de la Energía y demás Formas de Energía No Convencionales, PROURE, se definen sus objetivos, subprogramas y se adoptan otras disposiciones al respecto.

Sabogal, J. \& Moreno, E. (2011). "Evaluación de los proyectos de Mecanismo de Desarrollo Limpio (MDL) presentados a la Junta Ejecutiva de la Convención Marco para Cambio Climático de las Naciones Unidas (UNFCCC) entre el 2004 y el 2008 bajo criterios de sostenibilidad." En: Revista de la Facultad de Ciencias Económicas, Universidad Militar Nueva granada, XIX (1): 125-140.

Sabogal, J., Moreno, E. \& Ortega, G. (2010). "Procesos de Certificación de proyectos de captura de gases efecto invernadero en los mercados internacionales de carbono". En: Revista Gestión y Ambiente, Universidad Nacional de Colombia, 12 (3): 7-19.

Unidad de Planeación Minero Energética (UPME) (2003). Formulación de un Programa Básico de Normalización para 
Aplicaciones de Energías Alternativas y Difusión - Utilización de la Energía Geotérmica Documento Descriptivo. UPME (eds.) Bogotá, Colombia.

Unidad de Planeación Minero Energética (UPME) (2007). Plan Energético Nacional 2006-2025 - Contexto y Estrategias. UPME (eds.) Bogotá, Colombia.

Unidad de Planeación Minero Energética (UPME) (2010a). Programa de Uso Racional y Eficiente de Energía y Fuentes No Convencionales (PROURE) - Plan de Acción Indicativo 20102015. UPME (eds.) Bogotá, Colombia.
Unidad de Planeación Minero Energética (UPME) (2010b). Formulación de un Plan de Desarrollo para las Fuentes No Convencionales de Energía en Colombia (PDFNCE). UPME (eds.) Bogotá, Colombia.

Unidad de Planeación Minero Energética (UPME); Instituto de Hidrología, Meteorología y Estudios Ambientales (IDEAM); Departamento Administrativo de Ciencia, Tecnología e Innovación (COLCIENCIAS) \& Universidad Industrial de Santander (UIS) (2009). Atlas del Potencial Energético de la Biomasa Residual en Colombia. UPME (eds.) Bogotá, Colombia. 